\title{
Electrochemical capacitance of a leaky nanocapacitor
}

\author{
Xuean Zhao and Jian Wang \\ Department of Physics, The University of Hong Kong, Pokfulam Road, Hong Kong, China \\ Hong Guo \\ Centre for the Physics of Materials and Department of Physics, McGill University, Montreal, PQ, Canda H3A 2T8
}

(Received 28 April 1999; revised manuscript received 13 July 1999)

\begin{abstract}
We report a detailed theoretical investigation on electrochemical capacitance of a nanoscale capacitor where there is a dc coupling between the two conductors. For this "leaky" quantum capacitor, we have derived general analytic expressions of the linear and second-order nonlinear electrochemical capacitance within a first-principles quantum theory in the discrete potential approximation. Linear and nonlinear capacitance coefficients are also derived in a self-consistent manner without the latter approximation and the self-consistent analysis is suitable for numerical calculations. At linear order, the full quantum formula improves the semiclassical analysis in the tunneling regime. At nonlinear order that has not been studied before for leaky capacitors, the nonlinear capacitance and nonlinear nonequilibrium charge show interesting behavior. Our theory allows the investigation of crossover of capacitance from a full quantum to classical regimes as the distance between the two conductors is changed. [S0163-1829(99)03548-1]
\end{abstract}

\section{INTRODUCTION}

The most significant development in electronic devices has been the progressive miniaturization of them: it is now common to fabricate electron device structures with dimensions at mesoscopic scale and even at nanoscale. One of the important directions in nanoelectronics research is to understand device properties that relate to the existence of small dimensions. In this work, we investigate the notion of electrochemical capacitance for conductors in the mesoscopic or nanoscale and the nonequilibrium charge distribution at the nonlinear level. Using a full first-principles quantum theory, a semiclassical theory, as well as a direct numerical solution, we construct an overall physical picture on the effects of quantum tunneling to the nanoscale capacitance. We also investigate the density of state correction to capacitance at nonlinear orders of the external bias. For a pair of very small conductors, it has been known that the capacitance may be quite different from the usual parallel plate capacitance formula given by $C_{o} \sim 1 / W$, where $W$ is the distance between the two plates. Apart from the usual electrostatic fringe effect, there are quantum corrections to the classical formula. Quantum corrections come from several sources: a finite density of states of the plates, a finite screening length to the electron-electron interactions, and quantum tunneling.

The quantum correction to classical formula due to density of states (DOS) has been theoretically ${ }^{1,2}$ and experimentally ${ }^{3}$ investigated in the literature by a number of authors. For semiconductor heterojunctions they found that DOS contributes a factor to the capacitance given by $C_{D O S}$ $=e^{2}(d N / d E)$ where $d N / d E$ is the total density of states of the system. Thus, the total capacitance $C$ is a result of a series connection of $C_{o}$ and $C_{D O S}: 1 / C=1 / C_{o}+1 / C_{D O S}$. This formula has been theoretically studied from a dynamic point of view and was derived in the low-frequency limit of an ac theory. ${ }^{4,5}$ Significantly, these investigations on DOS effects focused on the linear capacitance coefficient $C$, namely $C$ is the linear coefficient of the charge pile up on a capacitor plate as a function of the external bias voltage. Recently some attention ${ }^{6}$ has been paid to the nonlinear regime: due to the nonlinear bias dependence of local DOS there is also a nonlinear bias dependence of the nonequilibrium charge. The nonlinear capacitance coefficients is one of the topics that will be further investigated below.

Mesoscopic electrochemical capacitance has been found ${ }^{7}$ to obey, within a magnetic field, weaker Onsager-Casimir symmetry relations. For example it is no longer a symmetric function of magnetic field. ${ }^{7}$ The asymmetry of electrochemical capacitance has been observed for a gate close to the edge of a quantum Hall bar. ${ }^{8}$ The magneto-electrochemical capacitance of a three-dimensional quantum dot with three probes has been studied numerically in detail in Ref. 9. It is found that at low-magnetic fields the magnetocapacitance shows a large asymmetry under a magnetic field reversal. At higher fields the capacitance is dominated by AharonovBohm-type oscillations and the fluctuations of the asymmetry is somewhat reduced. For the III-V tunneling heterostructures, the contribution of the density of states on the magnetocapacitance is also studied. ${ }^{10}$ The investigation of the frequency dependent electrochemical capacitance for a parallel plate capacitor within the nonequilibrium Green'sfunction formalism show interesting oscillatory behavior, which is related to the retardation effect of the Maxwell equations. $^{11}$

As mentioned above, quantum tunneling effect changes the capacitance value as predicted by the classical formula. This effect was recently addressed using numerical analysis of atomic junctions. ${ }^{12}$ Numerical calculations ${ }^{12}$ of aluminum atomic junctions with tiny DOS showed that at small distances $W$, the electrochemical capacitance $C=C(W)$ actually increases with $W$, which is due to tunneling effect. One expects that at larger $W$ when tunneling effects is diminished, the capacitance would follow a crossover to the classical prediction. However, due to the very small DOS of the 

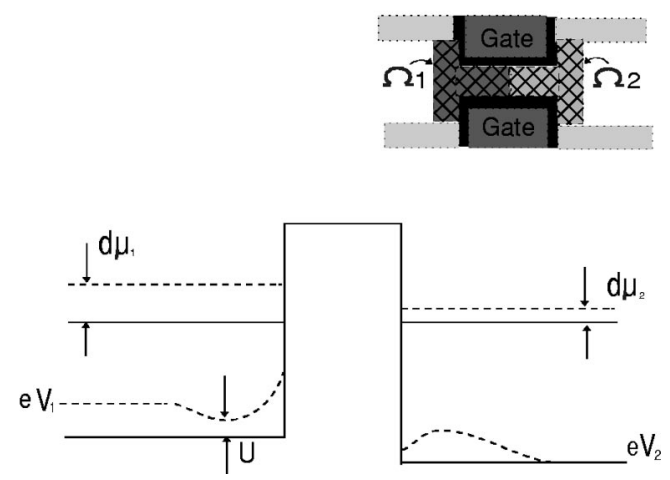

FIG. 1. The energy band configuration for a model barrier. Near the barrier the band bottom is different from that away the barrier. The inset is an ideal experimental setup of which the energy band is schematically shown in the figure.

atomic junction, ${ }^{12}$ no crossover to the classical formula was found in these atomic systems.

The correction to classical capacitance formula due to a finite screening length was most clearly demonstrated from a dynamic point of view on the electrochemical capacitance, due to the work of Christen and Büttiker ${ }^{13}$ where a conducting quantum-point contact (QPC) was found to establish a nonequilibrium charge resulting to a finite electrochemical capacitance. In particular they have derived a formula for a QPC with a semiclassical method, ${ }^{13}$

$$
\frac{R}{C}=\frac{1}{C_{o}}+\frac{1}{e^{2} \frac{d N_{1}}{d E}}+\frac{1}{e^{2} \frac{d N_{2}}{d E}},
$$

where $R$ is essentially a reflection probability of the QPC, $C_{o}$ is geometric capacitance, $d N_{1} / d E$ and $d N_{2} / d E$ are the total DOS in the regions to the left and to the right of the QPC. Qualitatively, the numerical data of the aluminum tunnel junction $^{12}$ were consistent with Eq. (1) in that $C$ is proportional to $R$. Formula (1) is termed "semiclassical" because not all the relevant scattering local partial density of states (LPDOS) were included in its derivation. The notion of scattering LPDOS was proposed by Büttiker ${ }^{4}$ and subsequently by Gasparian, Christen and Büttiker, ${ }^{14}$ and it plays a very important role in low frequency ac transport as well as nonlinear de transport. LPDOS describes the probability of various scattering processes. ${ }^{14}$ Consider a tunnel barrier as shown in Fig. 1. An example of a LPDOS is denoted by $d \sigma_{22}(\mathbf{r}) / d E$ which is the contribution of carriers at position $\mathbf{r}$ to the DOS, and these carriers come from region 2 and ultimately return to region 2 . Although region 2 is on the righthand side of the tunnel barrier (see Fig. 1), $d \sigma_{22}(\mathbf{r}) / d E \neq 0$ even when $\mathbf{r}$ is on the left-hand side of the barrier due to tunneling. In deriving ${ }^{13} \mathrm{Eq}$. (1) for a QPC, contributions such as $d \sigma_{22}(\mathbf{r}) / d E$ with position $\mathbf{r}$ on the other side of the QPC, has been neglected.

In this paper, we will further investigate nanoscale capacitors where the two conductors have a dc coupling, namely there is a dc "leakage" from one conductor to the other. For the linear electrochemical capacitance of a tunnel barrier, we improve formula (1) by including the tunneling contributions of various LPDOS. This way a full quantum capacitance formula is derived and will be compared with Eq. (1). For a single tunnel barrier there is a quantitative difference between these results in the quantum regime, and the difference diminishes as the classical limit is approached. The quantum formula and Eq. (1) allow investigations of a crossover from tunneling dominated regime to the classical regime, by varying the barrier width $W$. Our derivation as well as the derivation of Eq. (1) are within the discrete potential model ${ }^{15}$ that used an approximation where the space is coarse grained into a few regions. For the tunnel barrier they are regions to the left of the barrier (denoted by $\Omega_{1}$ ), to the right of the barrier $\left(\Omega_{2}\right)$, and the barrier region. To confirm that this approximation does not affect the predictions qualitatively, we have carried out extensive numerical calculations of the LPDOS by directly solving them without the approximation.

Recently, the theory of non-linear electrochemical capacitance has been formulated using the response theory. ${ }^{16}$ The electrochemical capacitance of a parallel plate capacitor is a nonlinear function of the bias voltage due to the finite DOS near the plates as mentioned above. In this paper, we will study effect of screening on the nonlinear electrochemical capacitance for the "leaky" capacitor, which is an important problem not investigated before and is relevant for experiments of scanning capacitance microscopy ${ }^{17}$ applied to nanosystems. We will derive a general expression of the second and third-order nonlinear quantum electrochemical capacitance using the discrete potential model. ${ }^{15}$ Our analysis naturally deduces, in appropriate approximations, a semiclassical expression of the second-order nonlinear electrochemical capacitance for QPC. Finally, to compare with results of the discrete potential model and semiclassical result, we have directly solved the Poisson equation and calculated numerically the linear and the second-order nonlinear electrochemical capacitance as a function of barrier width of a single tunneling barrier.

The main results of our investigation are summarized in the following sections. In the next section, we present our theory of the nonlinear electrochemical capacitance where full quantum tunneling effect is taken into account. At the linear order, we compare the quantum formula with the semiclassical formula; and using scattering Green's functions we derive second and third-order nonlinear results. In Sec. III, we present numerical calculations which is compared with the theoretical analysis. Finally, the last section summarizes the main findings.

\section{THEORY}

In general a two-probe system can be considered as having three regions, a scattering region and two electrodes. This is illustrated in Fig. 1 where the scattering region includes the scattering potential barrier, and two electrodes are the regions to the left $\left(\Omega_{1}\right)$ and to the right $\left(\Omega_{2}\right)$ of the barrier. We are interested in the electrochemical capacitance of this system by including the full quantum effects. If we refer regions $\Omega_{1}$ and $\Omega_{2}$ as the two conductors of a capacitor, we are dealing with a "leaky" capacitor since the potential barrier provides a dc coupling between the conductors. Far away from the the regions, the system is connected to contacts which are viewed as large thermodynamic reservoirs, hence in the contacts the electron distributions are 
Fermi Dirac. When a voltage $V_{1}$ is applied at contact 1 and $V_{2}$ at contact 2, assume $V_{1}<0$, the electron energy band at contact 1 is changed by $d \mu_{1}=e V_{1}$ and at contact 2 by $d \mu_{2}=e V_{2}$. The relative electrochemical potential difference is thus $d \mu=d \mu_{1}-d \mu_{2}$ : due to $d \mu$ electrons are injected into the system. The force acting on electrons comes from a combination of external and internal fields. In principle, motion of electrons in the total field can be solved by Schrödinger equation. In particular, we will adopt the scattering matrix approach formulated by Landauer, ${ }^{18}$ Imry, ${ }^{19}$ and Büttiker ${ }^{20,21}$ to solve the single-electron transport problem which gives the necessary LPDOS needed for the calculation of electrochemical capacitance.

Study of electrochemical capacitance is closely related to the calculation of changes of the local band $e U(\mathbf{r})$. It is clear that this local band change near the tunnel barrier is different from the shift $d \mu_{k}$, which occurs at the contacts far away from the barrier. At equilibrium conditions the electron energy near the barrier is given by $E_{t}=E+d \mu_{k}-e U(\mathbf{r})$ where $E$ is the electron energy at Fermi level without the applied voltage. $d \mu_{k}$ denotes the electrochemical potential change in reservoir $k$. Near the barrier electrons accumulate for regions where $E_{t}>E$ and deplete for regions where $E_{t}<E$. It is these accumulated charges which we must evaluate. The internal potential build-up $e U(\mathbf{r})$ can be solved by a self-consistent Poisson equation. For simplicity of discussion, in the following, we use $U_{1}(\mathbf{r})$ and $U_{2}(\mathbf{r})$ to denote this potential in regions $\Omega_{1}$ and $\Omega_{2}$, respectively. Furthermore, analytical derivation of capacitance formula in terms of microscopic quantities is possible if we use a space-averaged potential $U_{k}$ to replace the space-dependent potential $U_{k}(\mathbf{r})$, as was done in Ref. 13. This corresponds to the discrete potential model proposed by Christen and Buttiker. ${ }^{15}$

We represent the number of electrons in the region $\Omega_{k}$ $(k=1,2)$ incident from contact $\alpha(\alpha=1,2)$ by $\sigma_{k \alpha}$, which is a function of electron energy $E_{t}$. Hence $\sigma_{k \alpha}=\sigma_{k \alpha}(E$ $\left.+d \mu_{\alpha}-e U_{k}\right)$. The number of electrons without external bias (at equilibrium) is thus $\sigma_{k \alpha}(E)$, because $U_{k} \rightarrow 0$ when $d \mu \rightarrow 0$. By definition, the electrostatic (geometrical) capacitance $C_{o}$ between the two regions $\Omega_{1}$ and $\Omega_{2}$ is given by $C_{o}=\Delta Q_{1} /\left(U_{1}-U_{2}\right)$ [or by $\left.C_{o}=\Delta Q_{2} /\left(U_{2}-U_{1}\right)\right]$ where $\Delta Q_{k}(k=1,2)$ is the charge measured from the equilibrium value in region $\Omega_{k}$ regardless where they have come from, i.e., $\Delta Q_{k}=\Sigma_{\alpha}\left[\sigma_{k \alpha}\left(E_{t}\right)-\sigma_{k \alpha}(E)\right]$, where, to avoid confusion we use $k=\mathrm{I}$, II to denote the regions from now on. Since there are two electrodes, i.e., $\alpha=1,2, \Delta Q_{k}$ thus consists of two parts. For example, in region $\Omega_{I}$ (i.e., $k=\mathrm{I}$ ), a part of $\Delta Q_{I}$ is due to electrons incident from electrode $\alpha=1$, which are scattered back to region $k=\mathrm{I}$. We denote this part of $\Delta Q_{I}$ by $\Delta N_{1}\left(\Omega_{I}\right)=\sigma_{I 1}\left(E+d \mu_{1}-e U_{1}\right)-\sigma_{I 1}(E)$. The second part of $\Delta Q_{I}$ comes from electrons launched at electrode $\alpha$ $=2$ but ended up in region $k=\mathrm{I}$, this part is expressed by $\Delta N_{2}\left(\Omega_{I}\right)=\sigma_{I 2}\left(E+d \mu_{2}-e U_{1}\right)-\sigma_{I 2}(E)$. Hence, $\Delta Q_{I}$ $=\Delta N_{1}\left(\Omega_{I}\right)+\Delta N_{2}\left(\Omega_{I}\right)$.

The above partition of local charge according to where it comes from can be equally applied to the scattering local partial density of states. ${ }^{14}$ Hence, for example, $d \sigma_{12}\left(\Omega_{I}\right) / d E$ is the LPDOS, which is the DOS for an electron incident from electrode 2 passing through region $\Omega_{I}$ and reaching electrode 1. Similarly, $d \sigma_{22}\left(\Omega_{I}\right) / d E$ is the LPDOS, which is the DOS for an electron incident from electrode 2 passing through region $\Omega_{I}$ and eventually returning to electrode $2 .^{22}$ Both of these LPDOS describe the tunneling process. This latter term is neglected for a semiclassical calculations and is nonzero for a quantum analysis, as emphasized in Ref. 14. They both contribute to the electrochemical capacitance, ${ }^{23}$ which is the experimentally measured capacitance defined by

$$
C_{\mu}=\frac{e Q_{1}}{d \mu_{1}-d \mu_{2}} .
$$

The rest of the paper is devoted to calculate $C_{\mu}$ including all the quantum effects discussed above.

Based on the above discussions, we can write down the following two equations ${ }^{6}$ for the classical geometrical capacitance. Using charges of region $\Omega_{I}$,

$$
\begin{aligned}
C_{o} \times\left(U_{1}-U_{2}\right)= & \sigma_{I 1}\left(E+d \mu_{1}-U_{1}\right)-\sigma_{I 1}(E) \\
& +\sigma_{I 2}\left(E+d \mu_{2}-U_{1}\right)-\sigma_{I 2}(E) .
\end{aligned}
$$

Using charges of region $\Omega_{I I}$,

$$
\begin{aligned}
C_{o} \times\left(U_{2}-U_{1}\right)= & \sigma_{I I 1}\left(E+d \mu_{1}-U_{2}\right)-\sigma_{I I 1}(E) \\
& +\sigma_{I I 2}\left(E+d \mu_{2}-U_{2}\right)-\sigma_{I I 2}(E) .
\end{aligned}
$$

Because the same charge defines electrochemical capacitance $C_{\mu}$ as given by Eq. (2), we have

$$
C_{o} \times\left(U_{1}-U_{2}\right)=C_{\mu} \times\left(d \mu_{1}-d \mu_{2}\right) .
$$

Finally, it is important to remember that the internal electrostatic potential $U_{k}$ is a function of the electrochemical potential at the reservoirs,

$$
U_{1}=U_{1}\left(\mu_{1}, \mu_{2}\right), \quad U_{2}=U_{2}\left(\mu_{1}, \mu_{2}\right) .
$$

In above equations we have set electron charge $e=1$ so that $d \mu_{\alpha}=V_{\alpha}$, which is the bias voltage at reservoir $\alpha$.

Equations (3), (4), and (5) are the fundamental equations that we will use to derive quantum corrections to $C_{o}$ at the linear and nonlinear orders. Because our theory is gauge invariant, without loss of generality we set $V_{1}=V$ and $V_{2}=0$ throughout the following analysis.

\section{A. Linear electrochemical capacitance formula}

As discussed above, a semiclassical formula of the linear electrochemical capacitance has been derived in Ref. 13 in the form of Eq. (1). In this section, we derive a full quantum formula.

Taking derivatives of Eqs. (3), (4), and (5) with respect to $V$, we obtain

$$
\begin{gathered}
C_{o}\left(\frac{d U_{1}}{d V}-\frac{d U_{2}}{d V}\right)=\frac{d \sigma_{I 1}}{d E_{1}}\left(1-\frac{d U_{1}}{d V}\right)-\frac{d \sigma_{I 2}}{d E_{2}} \frac{d U_{1}}{d V} \\
C_{o}\left(\frac{d U_{2}}{d V}-\frac{d U_{1}}{d V}\right)=\frac{d \sigma_{I I 1}}{d E_{3}}\left(1-\frac{d U_{2}}{d V}\right)-\frac{d \sigma_{I I 2}}{d E_{4}} \frac{d U_{2}}{d V} \\
C_{o}\left(\frac{d U_{1}}{d V}-\frac{d U_{2}}{d V}\right)=C_{\mu},
\end{gathered}
$$


where $\quad E_{1} \equiv E+V_{1}-U_{1}, \quad E_{2} \equiv E+V_{2}-U_{1}, \quad E_{3}=E+V_{1}$ $-U_{2}$, and $E_{4}=E+V_{2}-U_{2}$. In deriving the last equation, we have assumed that $C_{\mu}$ has no bias voltage dependence. ${ }^{24}$ In general the above derivatives should be done at a finite bias voltage $V$, but experimentally one can control this parameter and use very small voltages ${ }^{3} V \ll E$. Hence, we will evaluate the derivatives at the $V \rightarrow 0$ limit. In the above equations, the quantity $d \sigma_{k \alpha} / d E_{i}$ is just the LPDOS in the corresponding regions as discussed above (where we used the notation such as $\left.d \sigma_{I 1} / d E\right)$.

From Eqs. (7), (8), and (9), eliminating $d U_{1} / d V$ and $d U_{2} / d V$, we obtain

$$
\begin{aligned}
& \frac{\frac{d \sigma_{I 1}}{d E_{1}}}{\frac{\frac{d \sigma_{I 1}}{d E_{1}}+\frac{d \sigma_{I 2}}{d E_{2}}}{\frac{C_{\mu}}{d E_{3}}+\frac{d \sigma_{I I 2}}{d E_{4}}}} \\
& =\frac{1}{C_{o}}+\frac{1 \sigma_{I I 1}}{\frac{d \sigma_{I I 1}}{d E_{1}}+\frac{d \sigma_{I 2}}{d E_{2}}}+\frac{1}{\frac{d \sigma_{I I 1}}{d E_{3}}+\frac{d \sigma_{I I 2}}{d E_{4}}} .
\end{aligned}
$$

The electrochemical capacitance $C_{\mu}$ calculated from this formula is fully quantum: all the tunneling effects are taken into account through the appropriate LPDOS, which can be evaluated from quantum scattering calculations (see below).

The general result (10) can be reduced to the semiclassical form Eq. (1) if we apply the semiclassical version of the LPDOS. In the semiclassical limit, Ref. 13 showed that the LPDOS is given by

$$
\frac{d \sigma_{k \alpha}}{d E}=\sum_{\beta} D_{k}\left[\frac{T}{2}+\delta_{\beta \alpha}\left(R \delta_{\beta k}-\frac{T}{2}\right)\right]
$$

where $T$ is the transmission coefficient, $R$ is related to the reflection coefficient, $D_{I} \equiv d \sigma_{I 1} / d E_{1}+d \sigma_{I 2} / d E_{2}$ and $D_{I I}$ $\equiv d \sigma_{I I 1} / d E_{1}+d \sigma_{I I 2} / d E_{2}$ are essentially total local DOS in regions $\Omega_{I}$ and $\Omega_{I I}$. Substituting Eq. (11) into Eq. (10), it is straightforward to prove that Eq. (10) reduces to the result of Ref. 13

$$
\frac{R}{C_{11}}=\frac{1}{C_{o}}+\frac{1}{D_{I}}+\frac{1}{D_{I I}},
$$

where we used notation $C_{11}$ to denote the linear electrochemical capacitance $C_{\mu}$. If we further set $R=1$, i.e., no dc coupling is allowed between the two regions, formula (12) reduces to the familiar electrochemical capacitance of two plates where there is no dc current flowing through. ${ }^{5}$

In Sec. III, we will provide numerical plots of the full quantum and semiclassical formula, and compare them with direct numerical solution of the same problem, which does not employ the discrete potential model.

\section{B. Nonlinear electrochemical capacitance formula}

We now derive the second-order nonlinear electrochemical capacitance from the fundamental Eqs. (3), (4), and (5). A similar procedure leads to higher-order results. To obtain nonlinear results we expand Eqs. (3) and (4) order by order in terms of the bias voltage $V_{\beta}$ and internal potential $U_{\beta}$. The expansion coefficients are energy derivatives of the spectral function $\sigma_{k \alpha}$, where the first derivative is the linear LPDOS used in the last section, while the second derivative is the second-order nonlinear LPDOS, which is analyzed in the Appendix A. Similarly higher-order derivatives are the corresponding higher-order nonlinear LPDOS. To simplify notation in the following we denote $D_{k \alpha} \equiv d \sigma_{k \alpha} / d E$ and $\bar{D}_{k \alpha} \equiv d^{2} \sigma_{k \alpha} / d E$. $^{2}$

To second order in bias voltage, Eqs. (3) and (4) become

$$
\begin{gathered}
C_{0}\left(U_{1}-U_{2}\right)=\sum_{\beta} D_{I \beta} V_{\beta}-D_{I} U_{1}+\sum_{\beta} \frac{1}{2} \bar{D}_{I \beta}\left(V_{\beta}-U_{1}\right)^{2} \\
-C_{0}\left(U_{1}-U_{2}\right)=\sum_{\beta} D_{I I \beta} V_{\beta}-D_{I I} U_{2} \\
+\frac{1}{2} \sum_{\beta} \bar{D}_{I I \beta}\left(V_{\beta}-U_{2}\right)^{2}
\end{gathered}
$$

Using Eq. (11) and expression (A10) of Appendix A, in the semiclassical limit the above two equations become

$$
\begin{aligned}
C_{0}\left(U_{1}-U_{2}\right)= & D_{I}\left(\frac{T}{2}+R\right) V_{1}+D_{I} \frac{T}{2} V_{2}-D_{I} U_{1} \\
& +\frac{1}{2} R \bar{D}_{I}\left(V_{1}-U_{1}\right)^{2}+\frac{1}{2} T \bar{D}_{I}\left(V_{2}-U_{1}\right)^{2}
\end{aligned}
$$

and

$$
\begin{aligned}
-C_{0}\left(U_{1}-U_{2}\right)= & D_{I I} \frac{T}{2} V_{1}+D_{I I}\left(\frac{T}{2}+R\right) V_{2}-D_{I I} U_{2} \\
& +\frac{1}{2} T \bar{D}_{I I}\left(V_{1}-U_{2}\right)^{2}+\frac{1}{2} R \bar{D}_{I I}\left(V_{2}-U_{2}\right)^{2} .
\end{aligned}
$$

In terms of $C_{11}$ of Eq. (12), we obtain internal potential $U_{1}$ and $U_{2}$ to first order in voltage,

$$
U_{1}=R V_{1}+\frac{T}{2}\left(V_{1}+V_{2}\right)-\frac{C_{11}}{D_{I}}\left(V_{1}-V_{2}\right)
$$

and

$$
U_{2}=R V_{2}+\frac{T}{2}\left(V_{1}+V_{2}\right)+\frac{C_{11}}{D_{I I}}\left(V_{1}-V_{2}\right)
$$

Substituting Eqs. (17) and (18) into the quadratic terms of Eqs. (15) and (16), we obtain 


$$
\begin{aligned}
C_{0}\left(U_{1}-U_{2}\right)= & D_{I}\left(\frac{T}{2}+R\right) V_{1}+D_{I} \frac{T}{2} V_{2}-D_{I} U_{1} \\
& +\frac{1}{2} R \bar{D}_{I}\left(\frac{T}{2}+\frac{C_{11}}{D_{I}}\right)^{2}\left(V_{1}-V_{2}\right)^{2} \\
& +\frac{1}{2} T \bar{D}_{I}\left(R+\frac{T}{2}-\frac{C_{11}}{D_{I}}\right)^{2}\left(V_{1}-V_{2}\right)^{2}
\end{aligned}
$$

and

$$
\begin{aligned}
-C_{0}\left(U_{1}-U_{2}\right)= & D_{I I} \frac{T}{2} V_{1}+D_{I I}\left(\frac{T}{2}+R\right) V_{2}-D_{I I} U_{2} \\
& +\frac{1}{2} T \bar{D}_{I I}\left(R+\frac{T}{2}-\frac{C_{11}}{D_{I I}}\right)^{2}\left(V_{1}-V_{2}\right)^{2} \\
& +\frac{1}{2} R \bar{D}_{I I}\left(\frac{T}{2}+\frac{C_{11}}{D_{I I}}\right)^{2}\left(V_{1}-V_{2}\right)^{2}
\end{aligned}
$$

Combining the above two equations, we finally arrive at

$$
C_{0}\left(U_{1}-U_{2}\right)=C_{11}\left(V_{1}-V_{2}\right)+\frac{1}{2} C_{111}\left(V_{1}-V_{2}\right)^{2}
$$

with the nonlinear capacitance

$$
\begin{aligned}
C_{111}= & C_{11}\left[\frac{\bar{D}_{I}}{D_{I}}\left(\frac{T}{2}+\frac{C_{11}}{D_{I}}\right)^{2}-\frac{\bar{D}_{I I}}{D_{I I}}\left(\frac{T}{2}+\frac{C_{11}}{D_{I I}}\right)^{2}\right] \\
& +\frac{T}{R} C_{11}\left[\frac{\bar{D}_{I}}{D_{I}}\left(R+\frac{T}{2}-\frac{C_{11}}{D_{I}}\right)^{2}-\frac{\bar{D}_{I I}}{D_{I I}}\left(R+\frac{T}{2}-\frac{C_{11}}{D_{I I}}\right)^{2}\right] .
\end{aligned}
$$

This result indicates that the second order nonlinear electrochemical capacitance can be expressed in terms of microscopic quantities such as the various LPDOS as well as transmission and reflection coefficients. All of these are calculable and have been studied before. Hence this result is very useful in practical predictions of nonlinear capacitance coefficient, and it is valid even if there is a dc coupling between the two polarization regions of the conductor.

The general expression (22) is reduced in certain limiting situations. First, for a spatially symmetric system where $D_{I}$ $=D_{I I}$ and $\bar{D}_{I}=\bar{D}_{I I}$, Eq. (22) gives $C_{111}=0$. This is expected due to symmetry: since $C_{111}$ is the coefficient of the charge distribution expanded in second order of bias voltage [e.g., Eq. (21)], it must vanish as charge $Q \rightarrow-Q$ for symmetrical systems when $V \rightarrow-V$. Second, for a capacitor without dc coupling between the two conductors, i.e., for cases $T=0$ identically, Eq. (22) becomes

$$
C_{111}=C_{11}^{3}\left(\frac{\bar{D}_{I}}{D_{I}^{3}}-\frac{\bar{D}_{I I}}{D_{I I}^{3}}\right),
$$

which was first derived in a response theory. ${ }^{16}$ Finally, a point worthy some discussion is the "resonant transmission point' by setting $T=1$ and $R=0$. For this case from Eq. (12) the linear electrochemical capacitance $C_{11}=0$. But from Eq. (22) $C_{111} \neq 0$ and is given by

$$
C_{111}=\frac{1}{4}\left(\frac{\bar{D}_{I}}{D_{I}}-\frac{\bar{D}_{I I}}{D_{I I}}\right) \frac{1}{C_{0}^{-1}+D_{I}^{-1}+D_{I I}^{-1}},
$$

which is generally nonzero. Apparently, we would expect no charge accumulation when $T=1$ hence $C_{111}$ and all other capacitance coefficients would vanish. However, the $T=1$ limit in the above formula only states the fact that injected charges are going through from one capacitor plate to the other at the linear order, and it does not implicate the behavior of the charges at nonlinear order where in general $T$ $=T(E, U)$. Thus in setting $T(E)=1$ in Eq. (22) is not the true resonant transmission point: at nonlinear order the resonance occurs at $T(E, U)=1$.

\section{Analysis beyond discrete potential model}

So far, we have derived the linear and nonlinear electrochemical capacitance coefficients within the discrete potential model, in which the internal potential $U_{k}$ is parametrized in terms of a geometrical capacitance $C_{o}$. This parametrization is necessary in order to carried out analytical derivations, and it is adequate to reveal qualitative features of the physics. On the other hand, if one is willing to perform numerical calculations, it is possible to go beyond the discrete potential approximation. In this case, we can solve the internal potential $U=U(\mathbf{r})$ from a self-consistent Poisson equation. In this section, we derive capacitance expressions that are suitable beyond the discrete potential model.

We start from the charge pile up written as a threedimensional spatial integral of the charge density ${ }^{16}$

$$
Q_{\alpha}=\int_{\Omega_{\alpha}} \rho(x) d^{3} x \equiv \sum_{\beta} C_{\alpha \beta} V_{\beta}+\frac{1}{2} \sum_{\beta \gamma} C_{\alpha \beta \gamma} V_{\beta} V_{\gamma}+\cdots \text {. }
$$

Reference 6 has shown that charge density $\rho(x)$ is given in terms of the linear and nonlinear LPDOS, as

$$
\begin{aligned}
\rho(x)= & \sum_{\alpha} \frac{d \sigma_{\alpha}(x)}{d E}\left[V_{\alpha}-U(x)\right] \\
& +\frac{1}{2} \sum_{\alpha} \frac{d^{2} \sigma_{\alpha}(x)}{d E^{2}}\left[V_{\alpha}-U(x)\right]^{2}+\cdots
\end{aligned}
$$

To proceed further we must solve the internal Coulomb potential $U(x)$ by the Poisson equation

$$
-\nabla^{2} U(x)=4 \pi \rho(x) .
$$

As done previously, ${ }^{5,16}$ for perturbative analysis of the electrochemical capacitance we introduce the characteristic potential $u(x)$

$$
U(x)=\sum_{\alpha} u_{\alpha}(x) V_{\alpha}+\frac{1}{2} \sum_{\alpha \beta} u_{\alpha \beta} V_{\alpha} V_{\beta}+\cdots .
$$

Hence, instead of solving $U(x)$ we solve for $u(x)$ order by order. From Eqs. (26) to (28), we find Poisson like equations for the characteristic potentials ${ }^{26}$

$$
-\nabla^{2} u_{\alpha}+4 \pi \frac{d \sigma}{d E} u_{\alpha}=4 \pi \frac{d \sigma_{\alpha}}{d E}
$$


and

$$
-\nabla^{2} u_{\alpha \beta}+4 \pi \frac{d \sigma}{d E} u_{\alpha \beta}=4 \pi \frac{d \tilde{\sigma}_{\alpha \beta}}{d E},
$$

where $^{16}$

$$
\frac{d \tilde{\sigma}_{\alpha \beta}}{d E}=\frac{d^{2} \sigma_{\alpha}}{d E^{2}} \delta_{\alpha \beta}-\frac{d^{2} \sigma_{\alpha}}{d E^{2}} u_{\beta}-\frac{d^{2} \sigma_{\beta}}{d E^{2}} u_{\alpha}+\frac{d^{2} \sigma}{d E^{2}} u_{\alpha} u_{\beta} .
$$

With the help of Eqs. (29) and (30), the electrochemical capacitance can be calculated from the following expressions,

$$
\begin{gathered}
C_{\alpha \beta} \equiv \int_{\Omega_{\alpha}} Q_{\beta}(x) d^{3} x=\int_{\Omega_{\alpha}}\left(\frac{d \sigma_{\beta}}{d E}-\frac{d \sigma}{d E} u_{\beta}\right) d^{3} x \\
C_{\alpha \beta \gamma} \equiv \int_{\Omega_{\alpha}} Q_{\beta \gamma}(x) d^{3} x=\int_{\Omega_{\alpha}}\left(\frac{d \tilde{\sigma}_{\beta \gamma}}{d E}-\frac{d \sigma}{d E} u_{\beta \gamma}\right) d^{3} x
\end{gathered}
$$

where $Q_{\beta}(x)$ and $Q_{\beta \gamma}(x)$ are linear and nonlinear nonequilibrium charge distributions. These results are useful for numerical calculations where all the quantities on the righthand side can be obtained accurately. For instance Eq. (32) has been used in the analysis of atomic junctions. ${ }^{12}$ Equation (33) is derived here.

To end this section we note that in a numerical calculation, the LPDOS $d \sigma_{\alpha} / d E$ can be calculated using the scattering wave function ${ }^{27}$

$$
\frac{d \sigma_{\alpha}(x)}{d E}=\frac{1}{h \mathrm{v}}|\psi(x)|^{2},
$$

where $\mathrm{V}$ is the velocity of the carrier and $\psi(x)$ is the scattering wave function for incident wave coming from lead $\alpha$. Equations (29) and (30) can be numerically solved on a three-dimensional grid, for instance a multigrid technique was employed in Ref. 12 for such a purpose.

\section{NUMERICAL RESULTS}

In this section, we present numerical plots for electrochemical capacitance of the tunnel barrier in Fig. 1. The numerical curves were obtained along two lines: by plotting the analytical expressions (10), (12), and (22), which are within the discrete potential model; and by direct numerical solution of the self-consistent internal potential $U(\mathbf{r})$ and then applying expressions (32) and (33). ${ }^{28}$

To be specific, we choose a numerical calculation box with size $x_{L}-x_{R}=12 \lambda_{F}$ where $\lambda_{F}$ is Fermi wavelength of the scattering electron. Here, $x_{L, R}$ are the positions of left and right boundary of the calculation box. We fix the tunnel barrier of width $W$ symmetrically in the center of the calculation box. This way the regions $\Omega_{I}$ and $\Omega_{I I}$ discussed above are given by the space between the calculation box and the barrier walls. The quantum scattering problem by this single barrier is easily solved, from which we obtain various LPDOS using the scattering wavefunction according to Eq. (34). To apply expressions (10), (12), and (22), which are appropriate for the discrete potential model, we spatially av- erage these LPDOS in the respective regions which gives us the corresponding quantities in these expressions. On the other hand, in applying expressions (32) and (33), which uses the full spatial dependent internal potential, the spatial integration range should be the Thomas-Fermi screening length ${ }^{5}$ as discussed in Appendix B. The screening length is determined $^{5}$ by solving the Poisson-like Eqs. (29) [and Eq. (30) in the nonlinear case]. From now on, we will use atomic units such that $\hbar=2 m=e=1$. In typical nanoscale systems ${ }^{29}$ with charge density $10^{15}$, Fermi wavelength $\lambda_{F} \sim 47 \mathrm{~nm}$. In the following, we use $\lambda_{F}$ to set the unit for length and choose Fermi energy $E_{F}=0.31$.

Figure 2 plots the linear electrochemical capacitance obtained from different approaches as a function of the barrier width $W$ for the fixed barrier height $H_{0}=0.8$ : (a). the electrochemical capacitance $C$ calculated numerically from Eq. (32) (solid line); (b). the analytic quantum electrochemical capacitance formula in the discrete potential approximation $C_{q}$ from Eq. (10) (dotted line); (c). the semiclassical electrochemical capacitance ${ }^{13} C_{s}$ of Eq. (12) (dashed line); (d). the effective classical geometric capacitance $C_{o} \sim 1 / W$ (dotdashed line). For very large the barrier width, it is clearly shown that all curves approach to the classical behavior $\sim 1 / W$ since quantum tunneling effect is negligible. For thin barriers where tunneling effect is significant, the behavior of electrochemical capacitances $C, C_{q}$, and $C_{s}$ are completely different from the classical regime. In this quantum regime, as one increases the barrier width, the electrochemical capacitance increases rather than decreases. This increasing behavior at very small $W$ is expected since tunneling tends to diminish charge polarization, thus $C \sim 0$ when $W \sim 0$. Hence, $C(W)$ should indeed start from small values and increase a bit before it goes down when $W$ is large enough.

To examine the DOS correction that is another quantum effect, we note that one can only separate out the geometrical effect from the DOS effect in the semiclassical limit [as in Eq. (12)], and in general these effects are mixed. Furthermore, in a discrete potential model all the quantities (both in quantum and semiclassical calculations) are spatially averaged, hence capacitances are underestimated. This is why both $C_{q}$ and $C_{s}$ curves are consistently below the full numerical solution $C$. Figure 2 shows some difference between the quantum result $C_{q}$ and semiclassical result $C_{s}$. To understand this difference we have plotted the partial DOS $d n_{11}\left(\Omega_{I I}\right) / d E$ (solid line) and $d n_{12}\left(\Omega_{I I}\right) / d E$ (dotted line) in the inset of Fig. 2. As expected, $d n_{11}\left(\Omega_{I I}\right) / d E$ goes to zero for large barrier widthes where the semiclassical theory is a good approximation. It is nonzero in the quantum tunneling regime for small barrier width. $d n_{11}\left(\Omega_{I I}\right) / d E$ is also numerically much less than $d n_{12}\left(\Omega_{I I}\right) / d E$. Hence, neglecting $d n_{11}\left(\Omega_{I I}\right) / d E$ in the semiclassical analysis gives a small difference between $C_{s}$ and $C_{q}$ in the tunneling regime (see Fig. 2 ). To further compare with the semiclassical result of QPC of Ref. 13, we have also examined the behavior of capacitance by varying the barrier height $H_{0}$ for a fixed barrier width $W$ : the results using Eqs. (32), (10), and (12) are, again, similar in the quantum regime and the same in the classical regime. When the barrier height $H_{0}$ is relatively small, the appearance of quantum mechanism leads to a correction for semiclassical electrochemical capacitance.

The physical behavior of second-order nonlinear electro- 


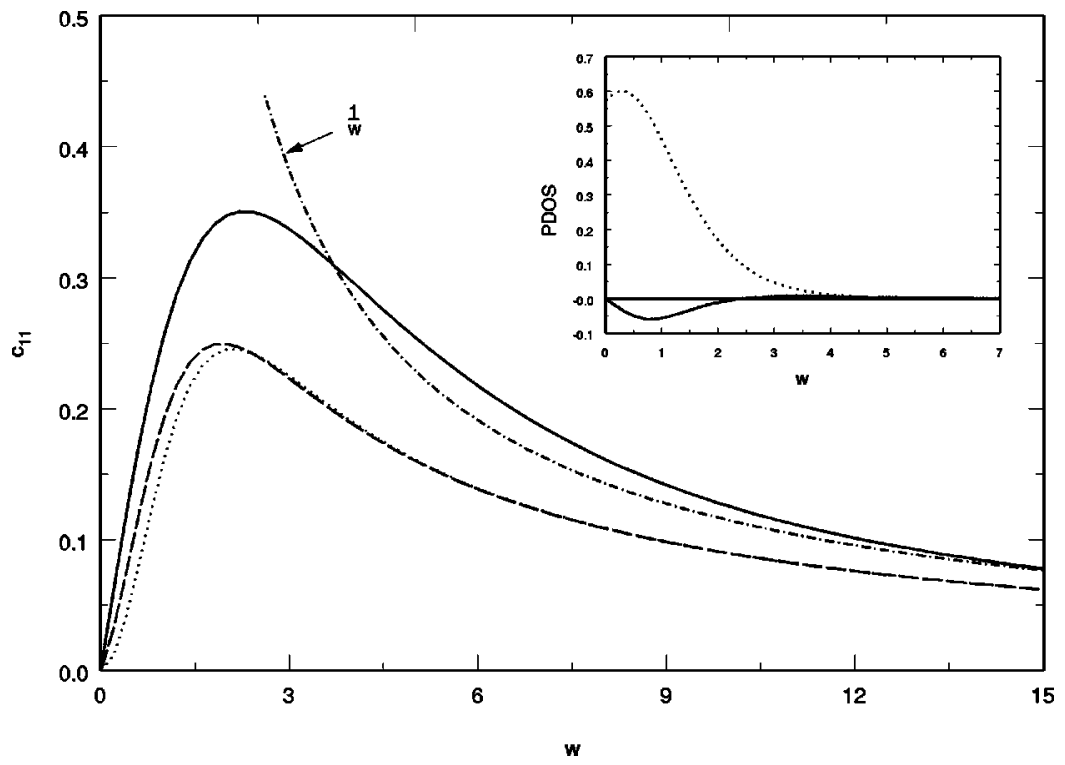

FIG. 2. The linear electrochemical capacitance as a function of barrier width $W$ for a square barrier with the barrier height $H_{0}=0.8$. The solid line is the full quantum numerical calculation $C$, the dashed line and dotted line are from the quantum result $C_{q}$ and the semiclassical result $C_{s}$ in the discrete potential approximation, respectively. The dot-dashed line is the classical result $C \sim 1 / W$. The inset: the corresponding partial density of states versus the barrier width $W$. The solid line is $d N_{11}\left(\Omega_{I I}\right) / d E$ and the dotted line is $d N_{12}\left(\Omega_{I I}\right) / d E$.

chemical capacitance coefficient $C_{111}$ can be studied for an asymmetric barrier: as discussed above $C_{111}=0$ for symmetric systems [see Eq. (22)]. For the asymmetric barrier where the barrier heights are $H_{1}=0.2$ and $H_{2}=1.0$, shown in the inset of Fig. 3(a), Fig. 3(a) plots $C_{11}$ versus $W$ and Fig. 3(b) plots $C_{111}$. The linear coefficient $C_{11}$ is very similar to that of Fig. 2 of a symmetric barrier. For the full quantum numerical result (solid line), $C_{111}$ starts from zero and becomes negative for very thin barrier, reaches minimum at around $W=1.0$, and then oscillates around zero. The oscillatory behavior can be traced to oscillations in second-order DOS $\bar{D}$ $=d^{2} N / d E^{2}$ of Eq. (22). In the inset of Fig. 3(b), we plot PDOS $D_{I}$ and $\bar{D}_{I}$. Correlating the behavior of $C_{111}$ and PDOS, it is clear that the negative values of $C_{111}$ is due to $\bar{D}$.

In Fig. 4, the linear and nonlinear nonequilibrium charge distribution for this asymmetric barrier, $Q_{1}(x)$ and $Q_{11}(x)$, are plotted. These quantities, especially $Q_{11}(x)$, have not been studied carefully before. It is thus interesting to offer several observations. (a) The linear charge distribution $Q_{1}(x)$ is in the form of a resistance dipole, ${ }^{25}$ whereas the nonlinear charge $Q_{11}(x)$ is more like a quadrupole. (b). The linear charge distribution is numerically much larger than the nonlinear charge distribution. The total charges are conserved, i.e., $\int Q_{1}(x) d x=\int Q_{11}(x) d x=0$. (c). In the discrete potential model, the average nonlinear charge $Q_{11}$ is numerically even smaller. Because of this spatial average, the nonlinear charge distribution becomes a dipole in the discrete potential model. This is responsible for the difference between full quantum calculation and that of the discrete potential model.

\section{DISCUSSION AND SUMMARY}

In this paper, we have investigated the quantum version of a "leaky capacitor" in the coherent nanoscale regime in both linear and nonlinear order in terms of the external bias voltage. We have derived an analytic formula of electrochemical capacitance where the two plates have a dc coupling, and tunneling effects between the two plates are fully included by explicitly using partial local density of states $d n_{\alpha \alpha}\left(\Omega_{k}\right) / d E$. Within the discrete potential model where all quantities are averaged over the polarization regions, analytic expressions for the linear and second-order nonlinear electrochemical capacitance have been derived. In addition, linear and nonlinear capacitance coefficients formula are derived in terms of the self-consistent potential, and these formula are suitable for numerical calculations in the full quantum regime. Our calculation showed that the analytic results using the discrete potential model are consistent with the full numerical solution, for the single tunnel barrier structure. The electrochemical capacitance formula derived in this paper are suitable for analyzing ac transport at relatively low frequency. At very high frequency, one has to consider radiation effect thus the quantum equation must be solved self consistently with the full Maxwell equation instead of the Poisson equation used here.

Quantum behavior of the electrochemical capacitance is found in the tunneling regime that the capacitance increases with the barrier width $W$. This is in clear contrast to the classical behavior of $1 / W$. What is the condition that this nonclassical phenomenon be observed? Let's consider this question using the semiclassical formula ${ }^{13} \mathrm{Eq}$. (12), which can be rewritten as

$$
C \approx \frac{R}{\frac{1}{C_{o}}+\frac{1}{D}}
$$

For tunneling, $R \approx[1-\exp (-W / l)]$ where $l$ is a characteristic length depending on system details such as the barrier heights. When $C=C(W)$ increases with $W$, we have $\partial C / \partial W>0$, which gives to a condition for the range of $W$ that allows the nonclassical behavior. Using Eq. (35), for tiny capacitor plates $D \ll C_{o}$, one can have a reasonable and experimentally accessible range of order $l$. On other hand, for large plates $D \gg C_{o}$, one can not observe the nonclassical effect unless $W$ is several orders smaller than $l$, which is not experimentally accessible. Hence, the condition to observe the non-classical behavior is tunneling and also small DOS. Systems which satisfy these conditions are nanoscale capaci- 

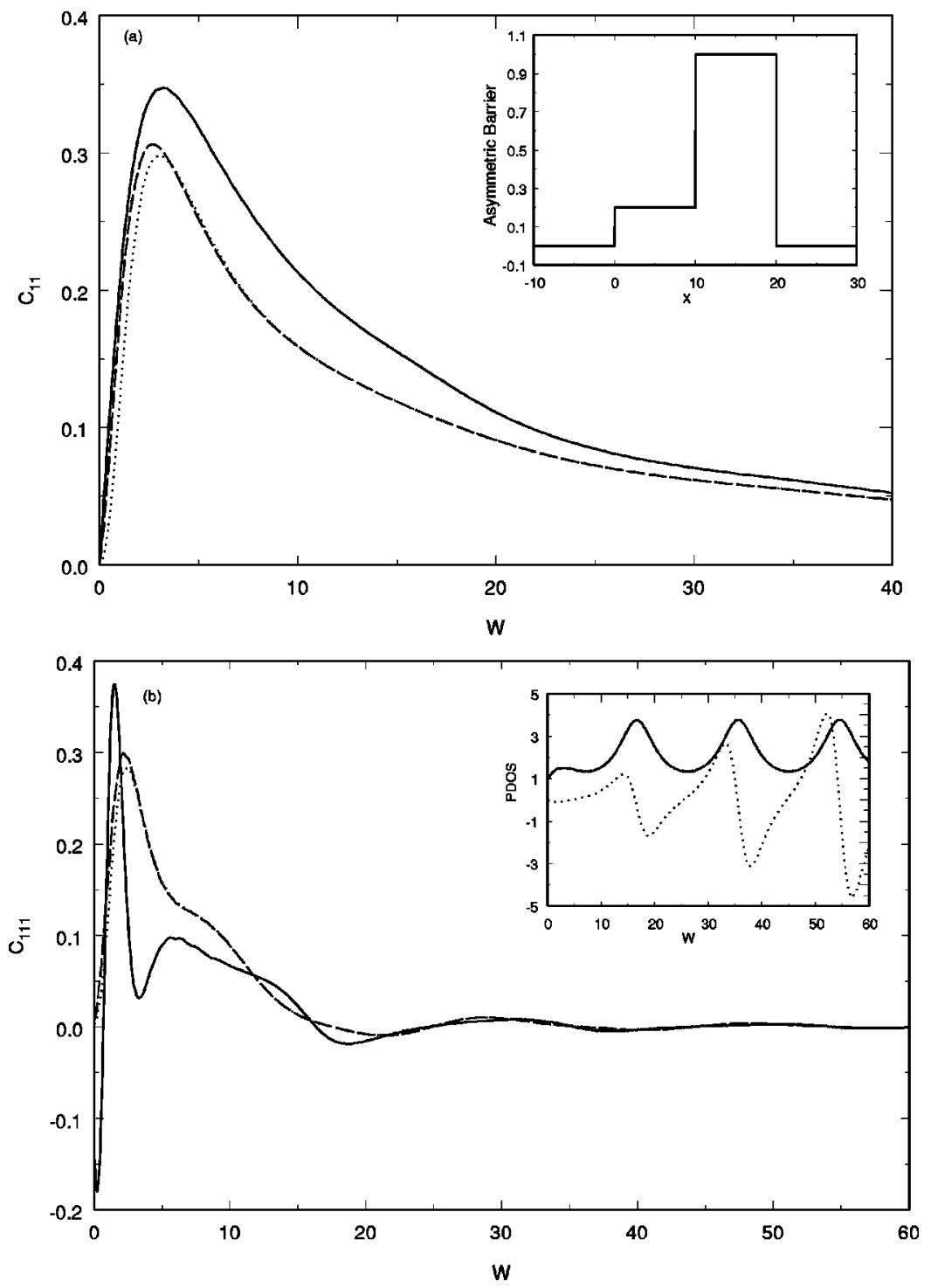

FIG. 3. (a) The linear electrochemical capacitance as a function of barrier width $W$ for the asymmetric barrier (see inset). The system parameters are $W_{1}=W_{2}, H_{1}=0.2, H_{2}=1.0$. (b). The second-order nonlinear electrochemical capacitance versus $W$. In (a) and (b), the solid, dashed, and dotted lines correspond to $C, C_{q}$, and $C_{s}$, respectively. In (b), we have multiplied $C_{q}$ and $C_{s}$ by a factor of 5 . The inset of (b): the corresponding partial DOS $D_{I}$ (solid line) and $\bar{D}_{I}$ (dotted line) as a function of $W$. For illustrating purpose, we have divided $\bar{D}_{I}$ by a factor of 30 . tors, whereas capacitors with large plates such as Josephson junctions (macroscopic) do not satisfy the DOS condition.

The nonlinear theory developed here can be pushed to higher order analytically within the discrete potential model.
At linear order the full quantum formula (10) and the semiclassical formula (12) give certain numerical difference in the quantum regime. There is a more substantial numerical difference between the discrete potential model and the full

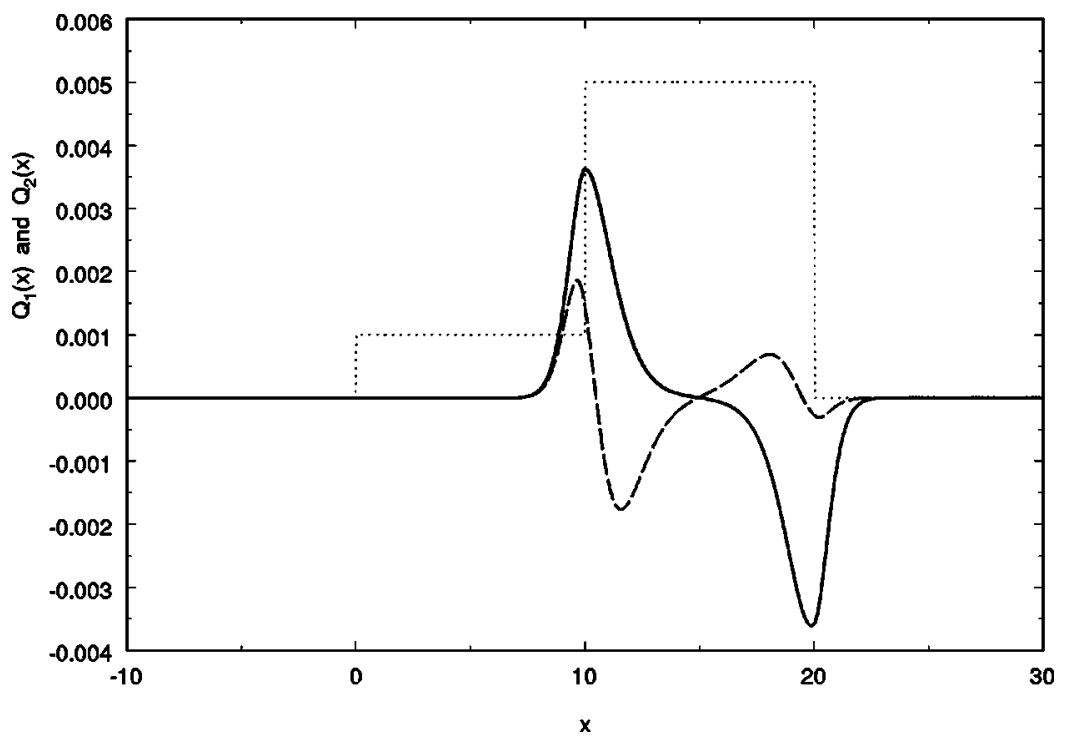

FIG. 4. The linear (solid line) and nonlinear (dashed line) charge distribution for the asymmetric barrier, where $W=20, H_{1}=0.2$, and $H_{2}$ $=1.0$. The dotted line shows the shape of asymmetric barrier. 
self-consistent numerical calculation using expressions (32) and (33), although all these results are qualitatively consistent. At second nonlinear order, the nonequilibrium charge distribution behaves as a quadrupole, this is compared to the resistance dipole when linear order charge is considered. It is interesting to note that the nonlinear charge can be nonzero when linear charge is zero: this happens at the linear resonance point. The nonlinear capacitance coefficient also behaves quite differently from the its linear counterpart, as shown by its oscillatory behavior linked to the nonlinear LPDOS.

\section{ACKNOWLEDGMENTS}

We gratefully acknowledge the financial support by a RGC grant from the SAR Government of Hong Kong under Grant Number HKU 7115/98P. H.G. is supported by the NSERC of Canada and the FCAR of Québec. J.W. would like to thank National Center for Theoretical Physics where part of this work was completed for hospitality. Z.X.A. thanks Professor M. Büttiker for helpful communication concerning the physics of electrochemical capacitance. We thank the Computer Center of The University of Hong Kong for computer facilities.

\section{APPENDIX A}

The nonlinear electrochemical capacitance depends on the nonlinear LPDOS, as shown in Sec. II. In this appendix, we derive the nonlinear (2nd order) LPDOS using Green's functions. In particular, we have to relate the second-order LPDOS $d^{2} \sigma_{\alpha \beta} / d E^{2}$ to the total LDOS $d^{2} \sigma / d E^{2}$, where indices $\alpha, \beta$ label the leads.

We start from the definition of LPDOS expressed in terms of the Green's function, ${ }^{29}$

$$
\frac{d \sigma_{12}(x)}{d E}=\operatorname{Re}\left[2 \pi i\left(G^{r} \Gamma_{2} G^{a} \Gamma_{1} G^{r}\right)_{x x}\right]
$$

where $G^{r}$ is the retarded Green's function, $\Gamma_{\alpha}$ is the linewidth function, and $\operatorname{Re}[\ldots]$ denotes the real part of $[\ldots]$. Using relation $^{14}$

$$
G_{x x_{1}}^{r} G_{x_{2} x}^{r}=G_{x x}^{r} G_{x_{2} x_{1}}^{r},
$$

we have

$$
\begin{aligned}
\left(G^{r} M G^{r}\right)_{x x} & =\sum_{x_{1} x_{2}} G_{x x_{1}}^{r} M_{x_{1} x_{2}} G_{x_{2} x}^{r} \\
& =G_{x_{x}}^{r} \sum_{x_{1} x_{2}} G_{x_{2} x_{1}}^{r} M_{x_{1} x_{2}}=G_{x x}^{r} \operatorname{Tr}\left[G^{r} M\right]
\end{aligned}
$$

where $M$ is a matrix. Equation (A1) becomes,

$$
\begin{aligned}
\frac{d \sigma_{12}}{d E} & =-2 \pi \operatorname{Im}\left[G_{x x}^{r} \operatorname{Tr}\left(\Gamma_{2} G^{a} \Gamma_{1} G^{r}\right)\right] \\
& =\frac{i}{2 \pi}\left(G_{x x}^{r}-G_{x x}^{a}\right) T=\frac{T}{2} \frac{d \sigma}{d E},
\end{aligned}
$$

where $\quad T=\operatorname{Tr}\left(\Gamma_{2} G^{a} \Gamma_{1} G^{r}\right) /\left(4 \pi^{2}\right)$ is the transmission coefficient, ${ }^{25}$ which is a real quantity; $d \sigma / d E$ is the total local DOS.

Taking energy derivative of Eq. (A1), we obtain

$$
\begin{aligned}
\frac{d^{2} \sigma_{12}}{d E^{2}}= & 2 \pi \operatorname{Im}\left[\left(G^{r} G^{r} \Gamma_{2} G^{a} \Gamma_{1} G^{r}\right)_{x x}+\left(G^{r} \Gamma_{2} G^{a} G^{a} \Gamma_{1} G^{r}\right)_{x x}\right. \\
& \left.+\left(G^{r} \Gamma_{2} G^{a} \Gamma_{1} G^{r} G^{r}\right)_{x x}\right] .
\end{aligned}
$$

The first term of Eq. (A5) can be simplified using Eq. (A3) as follows,

$$
\begin{aligned}
\left(G^{r} G^{r} \Gamma_{2} G^{a} \Gamma_{1} G^{r}\right)_{x x} & =G_{x x}^{r} \operatorname{Tr}\left(G^{r} \Gamma_{2} G^{a} \Gamma_{1} G^{r}\right) \\
& =G_{x x}^{r} \operatorname{Tr}\left(G^{r}\right) \operatorname{Tr}\left(\Gamma_{2} G^{a} \Gamma_{1} G^{r}\right) \\
& =\frac{T}{4 \pi^{2}}\left(G^{r} G^{r}\right)_{x x} .
\end{aligned}
$$

The other two terms of Eq. (A5) can be simplified in a similar fashion. We thus have

$$
\begin{aligned}
\frac{d^{2} \sigma_{12}}{d E^{2}} & =\frac{T}{2 \pi} \operatorname{Im}\left[2\left(G^{r} G^{r}\right)_{x x}+\left(G^{r} G^{a}\right)_{x x}\right] \\
& =-\frac{i T}{\pi}\left[\left(G^{r} G^{r}\right)_{x x}-\left(G^{a} G^{a}\right)_{x x}\right]=T \frac{d^{2} \sigma}{d E^{2}} .
\end{aligned}
$$

In deriving the last equation we used the fact that $G^{r} G^{a}$ is a real quantity. Using Eq. (A7) we find

$$
\frac{d^{2} \sigma_{11}}{d E^{2}}+\frac{d^{2} \sigma_{22}}{d E^{2}}=\frac{d^{2} \sigma}{d E^{2}}-\frac{d^{2} \sigma_{12}}{d E^{2}}-\frac{d^{2} \sigma_{21}}{d E^{2}}=(1-2 T) \frac{d^{2} \sigma}{d E^{2}} .
$$

Now we consider a system with a dc current passing through. Due to polarization we again divide the system into two regions $\Omega_{I}$ and $\Omega_{I I}$. In the semiclassical treatment where the tunneling is neglected, the partial DOS $d^{2} \sigma_{22} / d E^{2}=0$ in region $\mathrm{I}$, and similarly $d^{2} \sigma_{11} / d E^{2}=0$ in region II. These relations and Eqs. (A7) and (A8) lead to

$$
\frac{d^{2} \sigma_{k \alpha \beta}}{d E^{2}}=\frac{d^{2} \sigma}{d E^{2}}\left\{T+\delta_{\alpha \beta}\left[(1-2 T) \delta_{\alpha k}-T\right]\right\}
$$

where $k$ labels the polarization region $\Omega_{k}$. For two probe system, it gives

$$
\frac{d^{2} \sigma_{k \alpha}}{d E^{2}}=\frac{d^{2} \sigma}{d E^{2}}\left[T+(1-2 T) \delta_{\alpha k}\right]
$$

This expression is the semiclassical second-order nonlinear LPDOS, which is in contrast to the semiclassical linear LPDOS Eq. (11) derived in Ref. 13. The nonlinear LPDOS plays a crucial role in determining the nonlinear electrochemical capacitance, as given in Sec. II.

\section{APPENDIX B}

In this appendix, we give an example of calculating the second-order nonlinear capacitance $C_{111}$ by directly solving 
Poisson equation. This can be done analytically only for very simple systems.

Consider a system which consists of three regions: two leads (regions I and III) and an infinite potential barrier (region II). Since the calculation is perturbative, we have to calculate the linear characteristic potential by solving Poisson equation Eq. (29). We assume that the partial local DOS $d \sigma_{1} / d E$ and $d^{2} \sigma_{1} / d E^{2}$ are constant in region I and zero otherwise. ${ }^{5}$ Similarly $d \sigma_{2} / d E$ and $d^{2} \sigma_{2} / d E^{2}$ are constant in region III and zero otherwise. The solution of the Poisson equation Eq. (29) is

$$
\begin{array}{cc}
\text { region I: } \quad u_{1}=1-A_{1} \exp \left(\frac{x}{\lambda_{1}}\right) \\
\text { region II: } \quad u_{1}=a_{1} x+b_{1} \\
\text { region III: } \quad u_{1}=A_{2} \exp \left(-\frac{x}{\lambda_{2}}\right),
\end{array}
$$

where $A_{1}, A_{2}, a_{1}$, and $b_{1}$ are constants to be determined. In Eq. (B1), we have defined the screening length $\lambda_{\alpha}^{-2}$ $=4 \pi d \sigma_{\alpha} / d E$ and the boundary conditions ${ }^{5}$ that $u_{1} \rightarrow 1$ as $x \rightarrow-\infty$ and $u_{1} \rightarrow 0$ as $x \rightarrow \infty$ have been used. Using the boundary condition that $u_{1}$ and $d u_{1} / d x$ be continuous at $x$ $=a / 2$ and $-a / 2$, it is straightforward to find

$$
\begin{gathered}
a_{1}=\frac{1}{a+\lambda_{1}+\lambda_{2}}, \quad b_{1}=\frac{a_{1}}{2}\left(a+2 \lambda_{2}\right) \\
A_{1}=a_{1} \lambda_{1} \exp \left(\frac{a}{2 \lambda_{1}}\right), \quad A_{2}=a_{1} \lambda_{2} \exp \left(\frac{a}{2 \lambda_{2}}\right)
\end{gathered}
$$

The linear electrochemical capacitance can be obtained immediately,

$$
\begin{aligned}
C_{11} & \equiv \int_{\Omega_{I}} \frac{\partial \rho(x)}{\partial V_{1}} d x=\frac{-1}{4 \pi} \int_{\Omega_{I}} \nabla^{2} u_{1} d x=\left.\frac{-1}{4 \pi} \nabla u_{1}\right|_{-a / 2} \cdot A \\
& =\frac{A}{4 \pi} \frac{1}{a+\lambda_{1}+\lambda_{2}},
\end{aligned}
$$

where $A$ is the cross-section area of the metallic wire. Using the global DOS $d N_{\alpha} / d E=$ Volume $d \sigma_{\alpha} / d E=\lambda_{\alpha} A d \sigma_{\alpha} / d E$ $=A / 4 \pi \lambda_{\alpha}$, we arrive at the result first obtained by Büttiker, ${ }^{5}$

$$
\frac{1}{C_{11}}=\frac{4 \pi a}{A}+\frac{1}{d N_{1} / d E}+\frac{1}{d N_{2} / d E} .
$$

With the solution of $u_{1}$, the Eq. (30) becomes

region I: $\quad-\nabla^{2} u_{11}+\frac{1}{\lambda_{1}^{2}} u_{11}=\frac{1}{\bar{\lambda}_{1}^{2}} A_{1}^{2} \exp \left(\frac{2 x}{\lambda_{1}}\right)$ region II: $\quad \nabla^{2} u_{11}=0$

region III: $\quad-\nabla^{2} u_{11}+\frac{1}{\lambda_{2}^{2}} u_{11}=\frac{1}{\bar{\lambda}_{2}^{2}} A_{2}^{2} \exp \left(\frac{2 x}{\lambda_{2}}\right)$, where we have introduced another screening length $\bar{\lambda}_{\alpha}^{-2}$ $=4 \pi d^{2} \sigma_{\alpha} / d E^{2}$ corresponding to LPDOS $d^{2} \sigma_{\alpha} / d E^{2}$ and $A_{1}$ and $A_{2}$ are known from the calculation of $u_{1}$. The solution of Eq. (B5) is

$$
\text { region I: } \quad u_{11}=-\frac{\lambda_{1}^{2}}{3 \bar{\lambda}_{1}^{2}} A_{1}^{2} \exp \left(\frac{2 x}{\lambda_{1}}\right)+B_{1} \exp \left(\frac{x}{\lambda_{1}}\right)
$$

region II: $u_{11}=a_{2} x+b_{2}$

$$
\text { region III: } \quad u_{11}=-\frac{\lambda_{2}^{2}}{3 \bar{\lambda}_{2}^{2}} A_{2}^{2} \exp \left(\frac{2 x}{\lambda_{2}}\right)+B_{2} \exp \left(\frac{x}{\lambda_{2}}\right)
$$

After matching boundary conditions at $x=a / 2,-a / 2$, we obtain

$$
B_{1} \exp \left(\frac{-a}{2 \lambda_{1}}\right)=\frac{\lambda_{1}}{\left(a+\lambda_{1}+\lambda_{2}\right)^{3}}\left[\frac{2 \lambda_{1}^{3} a+\lambda_{1}^{4}+2 \lambda_{1}^{3} \lambda_{2}}{3 \bar{\lambda}_{1}^{2}}+\frac{\lambda_{2}^{4}}{3 \bar{\lambda}_{2}^{-2}}\right] .
$$

The second-order nonlinear electrochemical capacitance $C_{111}$ is

$$
\begin{aligned}
C_{111} \equiv & \frac{1}{2} \int_{\Omega_{I}} \frac{\partial^{2} \rho(x)}{\partial V_{1}^{2}} d x \\
= & \left.\frac{-1}{4 \pi} \nabla u_{11}\right|_{-a / 2} \cdot A=\frac{\lambda_{1}}{6 \pi \bar{\lambda}_{1}^{2}} A_{1}^{2} \exp \left(-\frac{a}{\lambda_{1}}\right) \\
& -\frac{B_{1}}{4 \pi \lambda_{1}} \exp \left(-\frac{a}{2 \lambda_{1}}\right) \\
= & \frac{A}{4 \pi} \frac{1}{\left(a+\lambda_{1}+\lambda_{2}\right)^{3}}\left[\frac{\lambda_{1}^{4}}{3 \bar{\lambda}_{1}^{2}}-\frac{\lambda_{2}^{4}}{3 \bar{\lambda}_{2}^{2}}\right] .
\end{aligned}
$$

From the definition of the screening length, we have

$$
\frac{\lambda_{\alpha}}{A}=\frac{1}{4 \pi A \lambda_{\alpha} d \sigma_{\alpha} / d E}=\frac{1}{d N_{\alpha} / d E},
$$

where we have used the fact that there is charge polarization only in the region $A \lambda_{\alpha}$. Similarly, we obtain

$$
\frac{\bar{\lambda}_{\alpha}}{A}=\frac{1}{d^{2} N_{\alpha} / d E^{2}} .
$$

With the help of Eqs. (B9), (B10), and (B4), we finally have

$$
C_{111}=\frac{C_{11}^{3}}{3}\left[\frac{d^{2} N_{1} / d E^{2}}{\left(d N_{1} / d E\right)^{3}}-\frac{d^{2} N_{2} / d E^{2}}{\left(d N_{2} / d E\right)^{3}}\right],
$$

which agrees with Eq. (23). 
${ }^{1}$ F. Stern, Appl. Phys. Lett. 43, 974 (1983); Phys. Rev. B 5, 4891 (1972).

${ }^{2}$ S. Luryi, Appl. Phys. Lett. 52, 501 (1988).

${ }^{3}$ T.P. Smith, B.B. Goldberg, P.J. Stiles, and M. Heiblum, Phys. Rev. B 32, 2696 (1985); T.P. Smith III, W.I. Wang, and P.J. Stiles, ibid. 34, 2995 (1986).

${ }^{4}$ M. Büttiker, H. Thomas, and A. Prêtre, Phys. Lett. A 180, 364 (1993).

${ }^{5}$ M. Büttiker, J. Phys.: Condens. Matter 5, 9361 (1993).

${ }^{6}$ Due to the nonlinear bias dependence of the electrochemical capacitance, it was proposed that a quantum scanning capacitance microscopy is possible. See B.G. Wang, X.A. Zhao, J. Wang, and H. Guo, Appl. Phys. Lett. 74, 2887 (1999).

${ }^{7}$ T. Christen and M. Büttiker, Phys. Rev. B 55, R1946 (1997).

${ }^{8}$ W. Chen, T.P. Smith III, M. Büttiker, and M. Shayegan, Phys. Rev. Lett. 73, 146 (1994).

${ }^{9}$ H.Q. Wei, N.J. Zhu, J. Wang, and H. Guo, Phys. Rev. B 56, 9657 (1997); P. Pomorski, H. Guo, R. Harris, and J. Wang, ibid. 58, 15393 (1998).

${ }^{10}$ K.S. Chan, F. W. Sheard, G. H. Toombs, and L. Eaves, Phys. Rev. B 56, 1447 (1997).

${ }^{11}$ B.G. Wang, J. Wang, and H. Guo, Phys. Rev. Lett. 82, 398 (1999)

${ }^{12}$ J. Wang, H. Guo, J.L. Mozos, C.C. Wan, G. Taraschi, and Q.R. Zheng, Phys. Rev. Lett. 80, 4277 (1998).

${ }^{13}$ T. Christen and M. Büttiker, Phys. Rev. Lett. 77, 143 (1996).

${ }^{14}$ V. Gasparian, T. Christen, and M. Büttiker, Phys. Rev. A 54, 4022 (1996).

${ }^{15}$ T. Christen and M. Büttiker, Europhys. Lett. 35, 523 (1996).

${ }^{16}$ Z.S. Ma, J. Wang, and H. Guo, Phys. Rev. B 57, 9108 (1998); ibid. 59, 7575 (1999).

${ }^{17}$ R.C. Ashoori, H.L. Stormer, J.S. Weiner, L.N. Pfeiff, S.J. Pearton, K.W. Baldwin, and K.W. West, Phys. Rev. Lett. 68, 3088 (1992); P. Lafarge, H. Pothier, E.R. Williams, D. Esteve, C. Urbina, and M.H. Devoret, Z. Phys. B: Condens. Matter 85, 327 (1991); H. Tomiye and T. Yao, Appl. Phys. Lett. 69, 4050 (1996); C.J. Kang, C. K. Kim, J. D. Lera, Y. Kuk, K. M. Mang,
J. G. Lee, K. S. Suh, and C. C. Williams, ibid. 71, 1546 (1997); J.K. Leong, C.C. Williams, and J.M. Olson, Phys. Rev. B 56, 1472 (1997); B. Sll, C. Gatzke, and J.M. Fernandez, Semicond. Sci. Technol. 13, 423 (1998).

${ }^{18}$ R. Landauer, Philos. Mag. 21, 863 (1970).

${ }^{19}$ Y. Imry, in Directions in Condensed Matter Physics, edited by G. Grinstein and G. Mazenko (World Scientific, Singapore, 1986), p. 101.

${ }^{20}$ M. Büttiker, Phys. Rev. Lett. 57, 1761 (1986).

${ }^{21}$ M. Büttiker, IBM J. Res. Dev. 32, 317 (1988).

${ }^{22}$ The physical meaning should be understood in the semiclassical limit. The quantum value of $d n_{\alpha \alpha} / d E$ can be negative as shown in the inset of Fig. 2. For detailed discussion, see Ref. 14.

${ }^{23}$ Exactly the same discussion can be applied to the other two LPDOS at the tunneling situation, $d \sigma_{11}\left(\Omega_{I I}\right) / d E$ and $d \sigma_{21}\left(\Omega_{I I}\right) / d E$.

${ }^{24}$ For nanoscale conductors with very small DOS, its electrochemical capacitance can have a nonlinear voltage dependence due to sampling of different parts of the DOS as the bias is varied. If this is the case, we should then replace Eq. (9) by the following equation:

$$
C_{o}\left(\frac{d U_{1}}{d V}-\frac{d U_{2}}{d V}\right)=C_{\mu}+V \frac{d C_{\mu}}{d V} .
$$

${ }^{25}$ Here the Thomas-Fermi approximation has been assumed. For the discussion going beyond Thomas-Fermi approximation, see Y.D. Wei, B.G. Wang, J. Wang, and H. Guo, cond-mat/9902306 (unpublished).

${ }^{26}$ M. Büttiker, H. Thomas, and A. Prétre, Z. Phys. B: Condens. Matter 94, 133 (1994).

${ }^{27}$ L.P. Kouwenhoven, G. Schön, and L.L. Sohn, in Mesoscopic Electron Transport, edited by L.L. Sohn, L.P. Kouwenhoven, and G. Schön (Kluwer Academic Publishers, Dordrecht, 1997).

${ }^{28}$ S. Datta, Electronic Transport in Mesoscopic Systems (Cambridge University Press, New York, 1995).

${ }^{29}$ T. Gramespacher and M. Büttiker, Phys. Rev. B 56, 13026 (1997). 